\title{
Centralities in High Order Networks
}

\author{
Klaus Wehmuth, Artur Ziviani \\ ${ }^{1}$ National Laboratory for Scientific Computing (LNCC) \\ Av. Getúlio Vargas, 333, Quitandinha - CEP 25651-075 - Petrópolis, RJ - Brazil \\ $\{$ klaus, ziviani\}elncc.br
}

\begin{abstract}
We propose a method for computing centralities based on shortest paths in time-varying, multilayer, and time-varying multilayer networks using MultiAspect Graphs (MAG). Thanks to the MAG abstraction, these high order networks are represented in a way that is isomorphic to a directed graph. We then show that well-known centrality algorithms can be adapted to the MAG environment in a straightforward manner. Moreover, we show that, by using this representation, pitfalls usually associated with spurious paths resulting from aggregation in time-varying and multilayer networks can be avoided.
\end{abstract}

\section{Introduction}

In this paper, we discuss notions of centrality in high order networks. A high order network is any network that has additional structures, such as, layers, time representations, or any other structure with similar properties. A high order network is not restricted to have only one of the mentioned structures, as it is possible to create networks that are layered and time-varying, or even layered networks with multiple time structures representing a multi-scale time structure. In this paper, we represent high order networks by means of MultiAspect Graphs (MAGs) [Wehmuth et al. 2016, Wehmuth et al. 2017]. MAGs are an abstraction that can be used to represent time-varying, multilayer, combined time-varying-multilayer, or even higher order graphs. In a MAG, each independent structure of a high order network is represented by an aspect. In a MAG, aspects are used to represent the vertices set, layer set, time instants set, and so on. In [Wehmuth et al. 2016], MAGs are shown to be isomorphic to an object composed of a directed graph and one tuple. Algebraic representations and basic algorithms for MAGs are investigated in [Wehmuth et al. 2017]. These previous works pave the way for the notions of centralities in high order networks introduced here. In particular, we focus on shortest path centralities, since they are widely applied on complex network analysis and relatively straightforward to interpret. Moreover, we show that by using the proposed method, pitfalls usually associated with aggregation in time-varying and multilayer networks can be avoided. These aggregation side-effects are well-known in the literature of multilayer/time-varying networks [Pan and Saramäki 2011, Nicosia et al. 2012, Ribeiro et al. 2013, Kivela et al. 2014, De Domenico et al. 2016] and are manifested as additional paths that do not exist in the original network, or as additional shortest paths that also do not exist in the original networks. The presence of these artifacts on the aggregated network causes the shortest path based centrality algorithms to include these shortest paths into the calculation, leading to spurious results that are not consistent to the original network. 


\section{MultiAspect Graphs (MAGs)}

We define a MAG as $H=(A, E)$, where $E$ is a set of edges and $A$ is a finite list of sets, each of which is called an aspect. Each aspect $\sigma \in A$ is a finite set, and the number of aspects $p=|A|$ is called the order of $H$. Each edge $e \in E$ is a tuple with $2 \times p$ elements. All edges are constructed so that they are of the form $\left(a_{1}, \ldots, a_{p}, b_{1}, \ldots, b_{p}\right)$, where $a_{1}, b_{1}$ are elements of the first aspect of $H, a_{2}, b_{2}$ are elements of the second aspect of $H$, and so on, until $a_{p}, b_{p}$ which are elements of the $p$-th aspect of $H$. As a matter of notation, we say that $A(H)$ is the aspect list of MAG $H$ and $A(H)[n]$ is the $n$-th aspect of MAG $H$. We also define the set

$$
\mathbb{V}(H)=\underset{n=1}{\mathrm{X}} A(H)[n],
$$

which is the Cartesian product of all the $p$ aspects of the MAG $H$. We call the elements $\mathbf{v} \in \mathbb{V}(H)$ composite vertices. Note that each composite vertex $\mathbf{v} \in \mathbb{V}(H)$ has the form $\left(a_{1}, \ldots, a_{p}\right)$. Therefore, there is a close relation between MAG edges and pairs of composite vertices, since $\left(a_{1}, \ldots, a_{p}, b_{1}, \ldots, b_{p}\right) \sim(\mathbf{v}, \mathbf{u})=\left(\left(a_{1}, \ldots, a_{p}\right),\left(b_{1}, \ldots, b_{p}\right)\right)$, so that $\mathbf{v}=\left(a_{1}, \ldots, a_{p}\right)$ and $\mathbf{u}=\left(b_{1}, \ldots, b_{p}\right)$. From this relation between MAG edges and pairs of composite vertices, it is possible to build a directed graph of composite vertices, which is shown to be isomorphic to the MAG in [Wehmuth et al. 2016].

As a consequence of the isomorphism between MAGs and a directed graph, we then define the function

$$
\begin{aligned}
g:(A(H), E(H)) & \rightarrow(\mathbb{V}(H), \mathbb{V}(H) \times \mathbb{V}(H)) \\
H & \mapsto(\mathbb{V}(H), \psi(E(H)),
\end{aligned}
$$

which maps every MAG $H$ to its isomorphic directed graph $g(H)$. Further, we define the set of functions

$$
\begin{aligned}
\pi_{i}: \mathbb{V}(H) & \rightarrow A(H)[i] \\
\left(a_{1}, a_{2}, \ldots, a_{p}\right) & \mapsto a_{i},
\end{aligned}
$$

which extracts the $i$-th element of a composite vertex tuple.

Note that by the definition of $g(H)$ in Expression 2, the vertices of the directed graph $g(H)$ are tuples with $2 \times p$ elements. It is also possible, if desired, to have a more traditional graph representation where the vertices are simply points of a set with no additional meaning. In this case, the directed graph has to be complemented by a companion tuple, which allows the association of each vertex with its original MAG tuple. This companion tuple is a tuple with $p$ elements, where each element $0<i \leqslant p$ is given as $|A(H)[i]|$, the number of elements of the $i$-th aspect. Further details regarding the construction and usage of the companion tuple of a MAG can be found in [Wehmuth et al. 2017].

\subsection{MAG sub-determination}

A MAG sub-determination is a generalization of the aggregation applied to high order networks, in which layers or time instants can be aggregated, resulting in a traditional graph. Since a high order network can be represented by a MAG with 2 or more aspects, 
a sub-determination can be performed in more ways than the classic aggregation. The formal definition of MAG sub-determination can be found in [Wehmuth et al. 2016]. As an example, consider a MAG with two aspects, where the first aspect is a set of geographic localities and the second aspect is a set of layers. Such structure could be used, for instance, to represent a multi-modal urban transportation system, where the layers represent the distinct transportation modes (e.g. Bus, Subway, Ferry) and geographic location represent the stations. A possible sub-determination of such MAG, can be the aggregation upon the stations, which is a classic directed graph.

\section{Centralities in high order networks}

In graph theory and network analysis, a centrality can be understood as an indicator of the relative importance of the vertices or edges on the graph under analysis. Formally, it follows that for a given graph $G=(V, E)$, a vertex centrality $C_{v}$ can be seen as a function from the set of vertices of a graph to a set of nonnegative real numbers, i.e.

$$
C_{v}: V(G) \rightarrow \mathbb{R}^{+} \bigcup\{0\},
$$

where $V(G)$ is the vertex set of the graph $G$. Note that Function 4 induces a linear order relation upon its domain, which can, in turn, be understood as a centrality notion. The adopted centrality function should then properly reflect the kind of vertex relative importance to be expressed by the centrality notion. Similarly, edge centralities may be defined to reflect the relative importance of an edge.

For instance, the vertex betweenness centrality [Freeman 1977] is defined as

$$
C_{B}(v)=\sum_{s, t \in V(G)} \frac{\sigma(s, t / v)}{\sigma(s, t)},
$$

where $V(G)$ is the the vertex set of the graph $G, s, t \in V(G)$ are vertices of the graph $\mathrm{G}, v \in V(G)$ is the vertex for which the centrality is calculated, $\sigma(s, t)$ is the number of shortest paths connecting vertices $s$ and $t$, and $\sigma(s, t / v)$ is the number of shortest paths from $s$ to $t$ which pass through vertex $v$. If calculated for each $v \in V(G)$, Equation 5 can be seen as a possible implementation of Function 4.

\subsection{Extending the centrality notion to MAGs}

The evaluation of edge centralities in a MAG can be done in a straightforward way using the MAG full representation. Since this representation is a traditional directed graph in which the vertices are a subset of the composite vertices set and it carries all the edges of the MAG, it thus preserves the topological properties (i.e., their adjacency properties). It follows that edge centralities can be computed using the same methods applied to traditional directed graphs.

However, the evaluation of vertex centralities in MAGs is more complex than in a traditional graph. The first difference to be considered is that a MAG can be sub-determined and at the limit any aspect element can become a vertex. Nevertheless, the full representation of a MAG, which is shown to be isomorphic to the MAG [Wehmuth et al. 2016], is a directed graph. Hence, vertex centralities in a MAG can be defined in terms of the composite vertices present on the MAG, as well as, in 
terms of any sub-determination of the composite vertices. Moreover, it is possible to find the shortest paths necessary for centrality calculation in the MAG full representation and performing the sub-determination in a later step. This assures that only the paths present in the full MAG representation will be considered in the centrality calculation, leading to the conclusion that the additional paths created by the sub-determination process will not be considered in the calculation.

\section{Conclusion}

In this paper, we discuss the implementation of shortest path based centralities for MAGs. Since MAGs are isomorphic to directed graphs added with a tuple, it follows that wellknown centrality algorithms can be adapted for the MAG environment. Further, due to the structure of MAGs, it follows that centralities can also be calculated in sub-determined form. We show that the sub-determined centrality algorithms use only valid paths present on the MAG, making them different from using the traditional (not sub-determined) algorithm upon a sub-determined MAG.

As with the well-known centrality algorithms for directed graphs, MAG centrality algorithms can be adapted in various ways, such as different distance definitions, restricted ranges, weighted graphs, and so on. We also show that these adaptations can be ported to MAG algorithms in a similar way as the traditional algorithms themselves are adapted for the MAG environment. Finally, as an additional contribution, we make a python implementation of the algorithms discussed in this paper publicly available. ${ }^{1}$

\section{Acknowledgements}

This work is partially funded by CAPES, CNPq, FAPERJ, and FAPESP.

\section{References}

De Domenico, M., Granell, C., Porter, M. A., and Arenas, A. (2016). The physics of multilayer networks. arXiv, pages 1-22.

Freeman, L. C. (1977). A Set of Measures of Centrality Based on Betweenness. Sociometry, 40(1):35 - 41 .

Kivela, M., Arenas, A., Barthelemy, M., Gleeson, J. P., Moreno, Y., and Porter, M. a. (2014). Multilayer networks. Journal of Complex Networks, 2(3):203-271.

Nicosia, V., Tang, J., Musolesi, M., Russo, G., Mascolo, C., and Latora, V. (2012). Components in time-varying graphs. Chaos, 22(2):023101.

Pan, R. and Saramäki, J. (2011). Path lengths, correlations, and centrality in temporal networks. Physical Review E, 84(1):1-10.

Ribeiro, B., Perra, N., and Baronchelli, A. (2013). Quantifying the effect of temporal resolution on time-varying networks. Scientific reports, 3:3006.

Wehmuth, K., Fleury, É., and Ziviani, A. (2016). On MultiAspect graphs. Theoretical Computer Science, 651:50-61.

Wehmuth, K., Fleury, E., and Ziviani, A. (2017). Multiaspect graphs: Algebraic representation and algorithms. Algorithms, 10(1).

\footnotetext{
${ }^{1}$ http://github.com/wehmuthklaus/MAG_Algorithms
} 